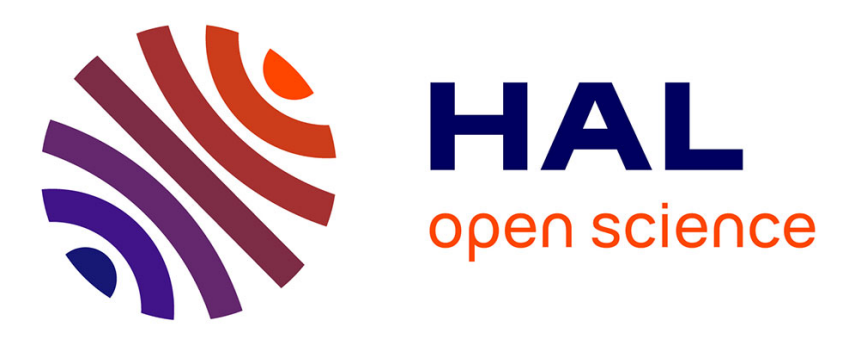

\title{
Conical Interfaces between Two Immiscible Fluids Induced by an Optical Laser Beam
}

\author{
A. Girot, J. Petit, R. Saiseau, T. Guérin, H. Chraibi, U. Delabre, Jean-Pierre \\ Delville
}

\section{- To cite this version:}

A. Girot, J. Petit, R. Saiseau, T. Guérin, H. Chraibi, et al.. Conical Interfaces between Two Immiscible Fluids Induced by an Optical Laser Beam. Physical Review Letters, 2019, 122 (17), 10.1103/PhysRevLett.122.174501 . hal-02915826

\section{HAL Id: hal-02915826 \\ https://hal.science/hal-02915826}

Submitted on 16 Aug 2020

HAL is a multi-disciplinary open access archive for the deposit and dissemination of scientific research documents, whether they are published or not. The documents may come from teaching and research institutions in France or abroad, or from public or private research centers.
L'archive ouverte pluridisciplinaire HAL, est destinée au dépôt et à la diffusion de documents scientifiques de niveau recherche, publiés ou non, émanant des établissements d'enseignement et de recherche français ou étrangers, des laboratoires publics ou privés. 


\title{
Conical Interfaces between Two Immiscible Fluids Induced by an Optical Laser Beam
}

\author{
A. Girot, J. Petit, R. Saiseau, T. Guérin, H. Chraibi, U. Delabre, and J. P. Delville \\ University of Bordeaux, CNRS, LOMA, UMR 5798, F-33405 Talence, France
}

(Received 14 December 2018; revised manuscript received 20 March 2019)

\begin{abstract}
We demonstrate the existence of conical interface deformations induced by a laser beam that are similar to Taylor cones in the electrical regime. We show that the cone morphology can be manipulated by fluid and laser parameters. A theory is proposed to quantitatively describe these dependences in good agreement with experimental data obtained for different fluid systems with low interfacial tensions. Counterintuitively, the cone angle is proved to be independent of the refractive index contrast at leading order. These results open a new optofluidic route towards optical spraying technology—an analogue of electrospraying — and more generally for the optical shaping of interfaces.
\end{abstract}

DOI:

One hundred years ago, in a pioneering work, Zeleny observed the destabilization of a suspended conducting liquid drop submitted to a sufficiently strong electric field $[1,2]$. The interface takes a conical shape, followed by a jet that usually breaks up into a spray of tiny droplets, a key phenomenon for electrospraying and electrospinning technologies [3-5]. Such conical menisci were theoretically understood by Taylor [6] and are now commonly termed as "Taylor cones." Beyond the surprising and fascinating elegance of such a simple conical solution for a complex mathematical problem involving deformable boundaries, this Taylor cone is important for applications. Indeed, the finite angle of the cone is a key parameter determining the size of the emitted jet, and thus of the resulting droplets [7].

Taylor cones are thus an essential component in processes as varied as the emission of monodisperse droplets [8], ink jet printing [3,5], the design of nanostructures [9] and encapsulation techniques [10]. Taylor cones were naturally generalized to electrically or magnetically induced deformations of interfaces between fluids presenting different conductivities, dielectric constants or magnetic susceptibilities [11]. Depending on these properties, but also on the nature of the field (either ac or dc) [12], the cone angle can vary over a wide range. Furthermore, conical shapes of fluid interfaces seem even more general, since portions of cones naturally appear in situations as varied as drops stretching [13], viscous breakup of pendant drops [14], tip streaming by Marangoni stress [15], or inertial jet eruption [16]. This suggests that various types of excitatory fields are able to induce conical deformations, as earlier suggested by Taylor himself [13]. In this context, considering the developments on the manipulation of fluids by light [17,18] and previous studies [19-21] where conical shapes could be suspected, a natural and surprisingly unresolved question is whether or not cones can be induced by light as well.

The goal of this Letter is to demonstrate the emergence of conical shapes in the optical regime and to characterize their geometry. Using very different fluid systems, we show indeed that above a critical radiation pressure exerted by a continuous laser wave, soft interfaces deform and adopt a conical shape. We propose a theory that correctly predicts the cone angles for a wide range of fluid and excitation parameters. Counterintuitively, we show that the cone angle does not depend on the refractive index contrast, while it is at the origin of the radiation pressure that induced the conical deformation.

To observe optically induced cones, we consider a continuous Gaussian laser wave that impinges a soft fluid interface from the liquid of higher refractive index as shown in Fig. 1(a). The laser beam is focused on the interface using standard optical elements that can be adjusted to vary the beam waist $w_{0}$ at the interface. At low power, the interface is gently deformed into a bell-shaped profile by optical radiation pressure [Fig. 1(a)]. This is due to the transfer of optical momentum of photons to the interface, as previously described [22,23]. Above a critical beam power $P_{c}$, the interface profile lengthens and sharpens, and a conical deformation emerges [Fig. 1(b)].

To characterize the geometry of the interface, we represent the local angle $\alpha_{i}$ in Fig. 1(c) as a function of the height $z$, i.e., the distance to the undeformed interface. This curve clearly exhibits a plateau region that is absent in the low power regime. This plateau demonstrates the existence of an optically induced conical deformation and defines its angle. To get insight in the mechanism at the origin of the cone formation, we image the optical path of the laser wave using specific optical filters [Fig. 1(d)]. The intense reflection of the laser beam at the cone interface tends to show that light is totally reflected inside the conical structure, which thus acts as a self-induced funnel guide. This is further confirmed in Fig. 1(c) by the fact that the incident angle $\alpha_{i}$ is always larger than the total reflection angle $\left[\alpha_{i}>\alpha_{\mathrm{TR}}=\arcsin \left(n_{1} / n_{2}\right)\right]$ in the plateau region. We anticipate that this total reflection condition is important to 


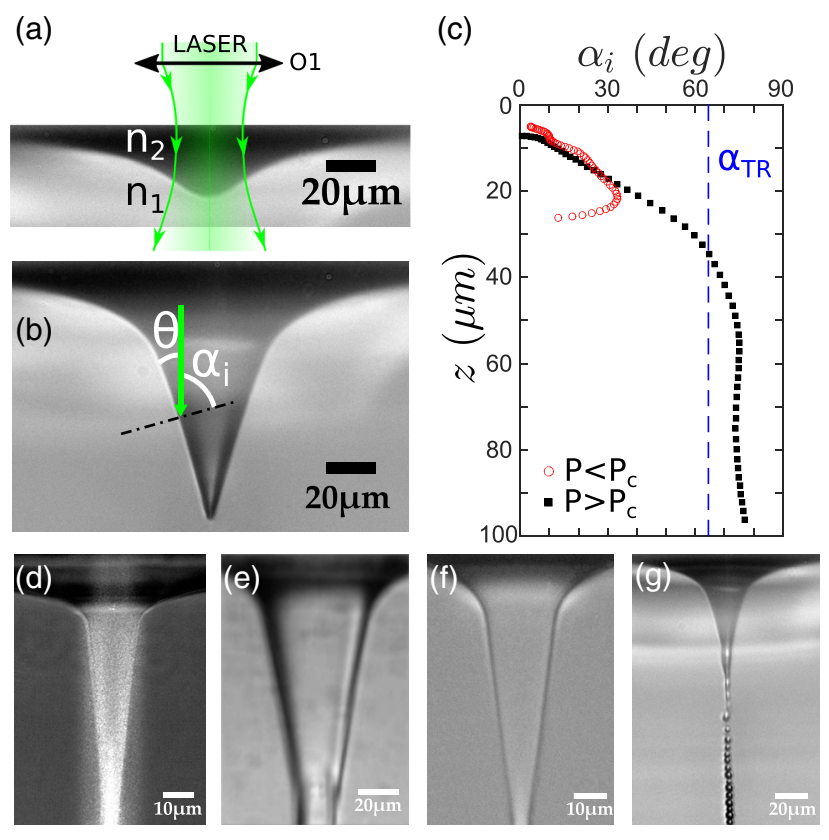

F1:1

F1:2

$\mathrm{F} 1: 3$

F1:4

F1:5

F1:6

$\mathrm{F} 1: 7$

$\mathrm{F} 1: 8$

F1:9

F1:10

$\mathrm{F} 1: 11$

F1:12

F1:13

F1:14

F1:15

F1:16

F1:17
FIG. 1. (a) Sketch of the experiment: a laser beam $(\lambda=532 \mathrm{~nm}$ in vacuum) is focused at the interface with the objective $\mathrm{O} 1$ (Olympus x10) and deforms this interface by radiation pressure. Deformation of the interface for $P<P_{c}$ (Winsor toluene $\mathrm{S} 1 \mathrm{~b}$ for $w_{0}=12.9 \mu \mathrm{m}, P=1.53 \mathrm{~W}$ ). (b) Conical deformation by radiation pressure for $P>P_{c}$ (Winsor toluene $\mathrm{S} 1 \mathrm{~b}$ for $w_{0}=12.9 \mu \mathrm{m}, P=1.55 \mathrm{~W}$ ). (c) Typical variation of the incident angle $\alpha_{i}$ as a function of the height of deformation for $P<P_{C}$ and $P>P_{C}$. Note that the curve for $P>P_{c}$ exhibits a clear plateau. $\alpha_{\mathrm{TR}}$ is the total reflection (TR) incident angle. (d) Light path revealing the total reflection mechanism inside the conical deformation (microemulsion S3). (e)-(g) Conical deformations for various experimental systems: (e) Winsor heptane S2 for $w_{0}=12.2 \mu \mathrm{m}, P=2.25 \mathrm{~W}$, (f) microemulsion $\mathrm{S} 3 \mathrm{e}$ for $w_{0}=$ $9.0 \mu \mathrm{m}$ and $P=2.89 \mathrm{~W}$, and $(\mathrm{g})$ jet and drop emission at the tip of the cone for Winsor toluene $\mathrm{S} 1 \mathrm{~b}$ system with $w_{0}=8.8 \mu \mathrm{m}$ and $P=1.06 \mathrm{~W}$.

explain the formation of optically-induced cones in our conditions.

To test the generality of optical liquid cones, we consider three main experimental fluid systems that are transparent at the used optical wavelength (optical absorption smaller than $3 \times 10^{-4} \mathrm{~cm}^{-1}$ ) and based on Winsor phases (toluene: S1a-S1b, heptane: S2) and quasicritical microemulsions (S3a-S3e). By varying chemical composition or temperature, we obtain in the end eight subsystems denoted by S1a-S1b, S2, S3a-S3e (see Supplemental Material [24-28] for details). This enables us to vary the refractive index contrast involved in the radiation pressure $\left(\Delta n=n_{2}-\right.$ $\left.n_{1}=0.0129-0.1449\right)$ and the interfacial tension involved in the restoring capillary pressure $\left(\gamma=2 \times 410^{-7}-1 \times\right.$ $310^{-5} \mathrm{~N} / \mathrm{m}$ ) over more than one order of magnitude. Refractive indexes for various systems were measured by standard refractometry methods, while interfacial tensions were determined by analyzing the viscous breakup dynamics

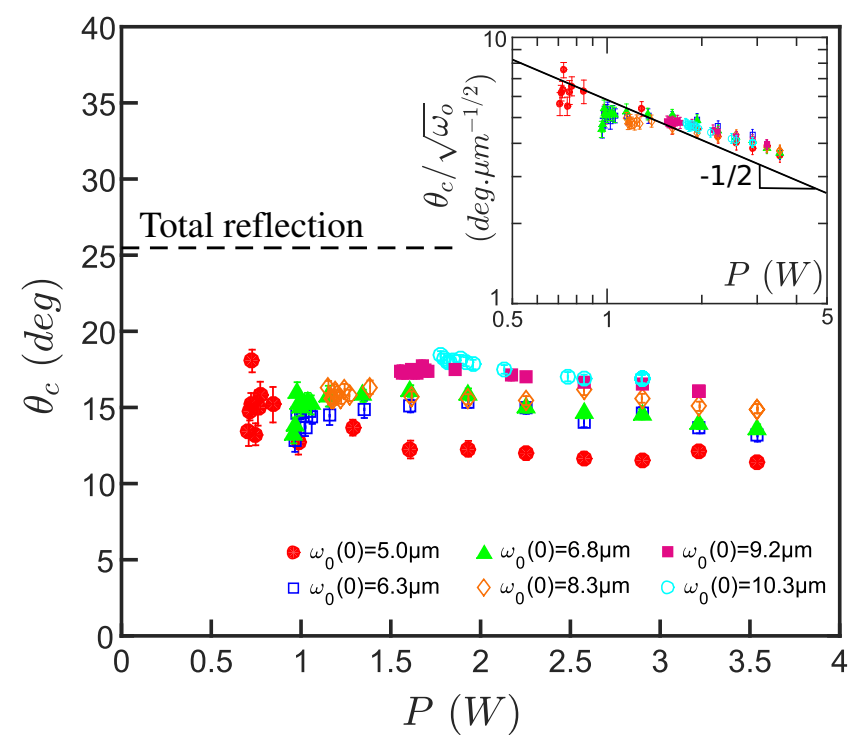

FIG. 2. Semiangle $\theta_{c}$ of the cone for Winsor toluene system $\mathrm{S} 1 \mathrm{~b}$ as a function of the power $P$ and the beam waist $w_{0}$ of the laser. $w_{0}(0)$ represents the laser beam waist extrapolated at zero power (see the Supplemental Material [24]). The dashline indicates the total reflection value $\pi / 2-\alpha_{\mathrm{TR}}$. Inset: Cone semiangle rescaled by $\sqrt{w_{0}}$ versus laser power $P$ in $\log$-log scale.

of liquid thread [29]. As illustrated in Fig. 1, stationary optically induced cones are generated for all experimental systems. Similar to electrified interfaces, these conical shapes are very stable and robust for both turbid (S1, S3) and nonturbid (S2) fluid systems. Remarkably, the conical structure often emerges together with a jet that emits droplets, as illustrated at the bottom in Fig. 1(g). Importantly, as shown in Fig. 1, we observe that the cone angle is specific to each fluid system, indicating that fluid properties are important to define the cone morphology.

We now quantify the effects of the laser parameters on the cone angle. The edge of the cone is detected by a homemade image analysis program that measures the cone semiangle $\theta_{c}=\pi / 2-\alpha_{i}$ in the plateau region [see Fig. 1(c)] after averaging over several stationary profile pictures. In Fig. 2, we show how $\theta_{c}$ depends on the incident laser power $P$ at various waists $w_{0}$ for system $S 1$ as an example. To be as accurate as possible, we note that increasing $P$ of our laser also results in an increase of the waist $w_{0}=g\left(P, w_{0}(0)\right)$ via a function $g$, which is fully characterized in the Supplemental Material [24], with $w_{0}(0)$ as the extrapolated waist at zero power. As shown in Fig. 2, the cone semiangle increases with the beam waist $w_{0}$ at a given power and slightly decreases with the applied power. This indicates that laser parameters are crucial for controlling the cone morphology.

To understand the physical mechanism at the origin of the conical deformation, it is useful to start with the force balance equation for an axi-symmetric stationary profile [22,30]:

$$
\gamma \kappa(r)-\Delta \rho g h(r)=\Pi_{\mathrm{rad}}(r)
$$




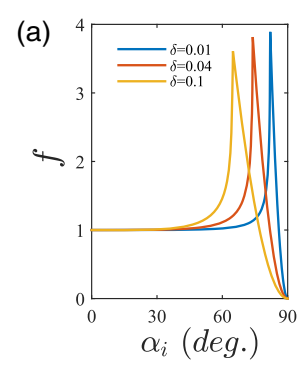

(c)

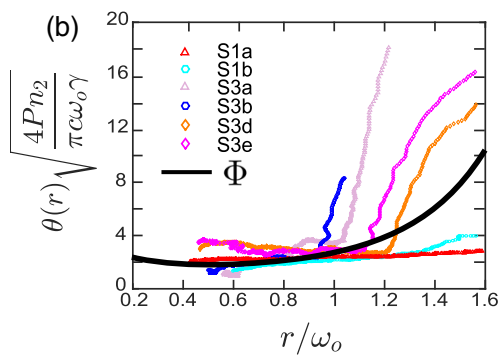

(d)

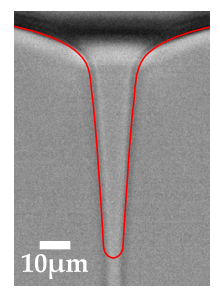

FIG. 3. (a) Variation of the $f$ function with the incident angle $\alpha_{i}$ for different relative index contrasts $\delta=2 . \Delta n /\left(n_{1}+n_{2}\right)$. (b) Variation of the normalized cone angle as a function of the normalized radial position $r / w_{0}$ for various liquid systems. The solid line indicates the theoretical prediction $\Phi(\cdot)$ [see Eq. (6)]. (c) Comparison between a theoretical cone deformation (red line) and an experimental deformation for microemulsion system S3b for $P=0.5 \mathrm{~W}$ and $w_{0}=5.8 \mu \mathrm{m}$. (d) Cone angle as a function of $\gamma / n_{2}$ for various systems and for a given ratio $w_{0} / P=4.57 \mu \mathrm{m} / \mathrm{W}$. The line indicates the theoretical prediction [Eq. (7)].

where both the Laplace pressure $\gamma \kappa(r)$ and buoyancy $\Delta \rho g h(r)$ balance the optical radiation pressure $\Pi_{\text {rad }}(r)$. Here, $r$ is the radial distance to the beam axis, $h$ is the height of the profile, $g$ the earth acceleration, and $\kappa$ is the local curvature. The optical radiation pressure is given for a continuous Gaussian wave $\left(\right.$ mode $\mathrm{TEM}_{00}$ ) by the following:

$$
\Pi_{\mathrm{rad}}(r)=\frac{n_{2}}{c} \frac{2 P}{\pi w_{0}^{2}} e^{-\frac{2 r^{2}}{w_{0}^{2}}} \delta f\left(\alpha_{i}\right)
$$
a geometric function that describes the variation of the radiation pressure with the local incident angle $\alpha_{i}$, $f\left(\alpha_{i}\right)=\cos ^{2}\left(\alpha_{i}\right)\left\{1+R\left(\alpha_{i}\right)-\left[\tan \left(\alpha_{i}\right) / \tan \left(\alpha_{t}\right)\right] T\left(\alpha_{i}\right)\right\} / \delta$, $R$ and $T$ being the reflexion and transmission Fresnel coefficients and $\alpha_{t}$ the refracted angle. This function $f$ is plotted in Fig. 3(a). Importantly, it displays a decreasing behavior above the total reflection angle $\alpha_{\mathrm{TR}}$, which means that the more inclined is the interface the less efficient is the radiation pressure. Therefore, above $\alpha_{\mathrm{TR}}$, the intensity of the radiation pressure is directly related to the local inclination of the interface, which will be the determinant to set the value of the cone angle.
We first describe the interface deformation at moderate beam power. As the optical Bond number (defined by using the beam waist as the characteristic length scale) Bo $=\left(\Delta \rho g w_{0}^{2} / \gamma\right) \approx 0.001-0.2$ is small, buoyancy can be neglected in first approximation. Furthermore, as the relative index contrast $\delta$ is also a small parameter, the radiation pressure can be considered as constant over a large range of inclination angles [i.e., $f \approx 1$, see Fig. 3(a)]. With these approximations, the force balance Eq. (1) at low powers becomes this:

$$
\frac{\gamma}{r} \frac{\partial}{\partial r}(r \cos \theta) \approx \frac{2 P \Delta n}{\pi c w_{0}^{2}} e^{-2 r^{2} / w_{0}^{2}}
$$

This equation is readily integrated for a closed profile, leading to $\cos \theta=\left(P \Delta n / 2 \pi c \gamma w_{0}\right)\left(1-e^{-2 u^{2}} / u\right)$ where $u=r / w_{0}$. The self-consistency condition that $\cos \theta$ remains lower than unity for all $r$ leads to the definition of a critical power

$$
P_{c} \approx 2.2 \frac{\pi c w_{0} \gamma}{\Delta n}
$$

above which one should observe strongly deformed interfaces, with inclination angles of the order of the total reflection angle. This condition is compatible with previous analyses [21,31] and is also in good agreement with the critical power values measured in our experiments (see Fig. S3 in the Supplemental Material [24]).

Above the critical power $P_{c}$, a new region appears where total reflection conditions hold, so that $f \approx 2 \theta^{2} / \delta$, indicating that the radiation pressure depends on the local profile slope. Moreover, in this region the opening angles $\theta$ are small compared to one, a condition which is satisfied in all our experiments. Hence, in this region the force-balance equation can be considerably simplified and becomes

$$
\frac{\gamma}{r}=\theta^{2} \frac{4 n_{2} P}{\pi c w_{0}^{2}} e^{-2 r^{2} / w_{0}^{2}}
$$

leading to:

$$
\theta(r)=\sqrt{\frac{\pi c \gamma w_{0}}{4 P n_{2}}} \Phi\left(\frac{r}{w_{0}}\right), \quad \Phi(X) \equiv \frac{e^{X^{2}}}{\sqrt{X}} .
$$

The local angle in the total reflection region is therefore proportional to the dimensionless function $\Phi(\cdot)$, which is plotted in Fig. 3(b). It exhibits a clear plateau characterizing the conical deformation in the range $r / w_{0} \approx 0.3-1$. To fully predict the cone angle variation with physical parameters, we characterize the minimal half-opening angle in Eq. (6), which is obtained for $r / w_{0}=0.5$. We find this:

$$
\theta_{c}^{*}=\beta \sqrt{\frac{c w_{0} \gamma}{P n_{2}}},
$$




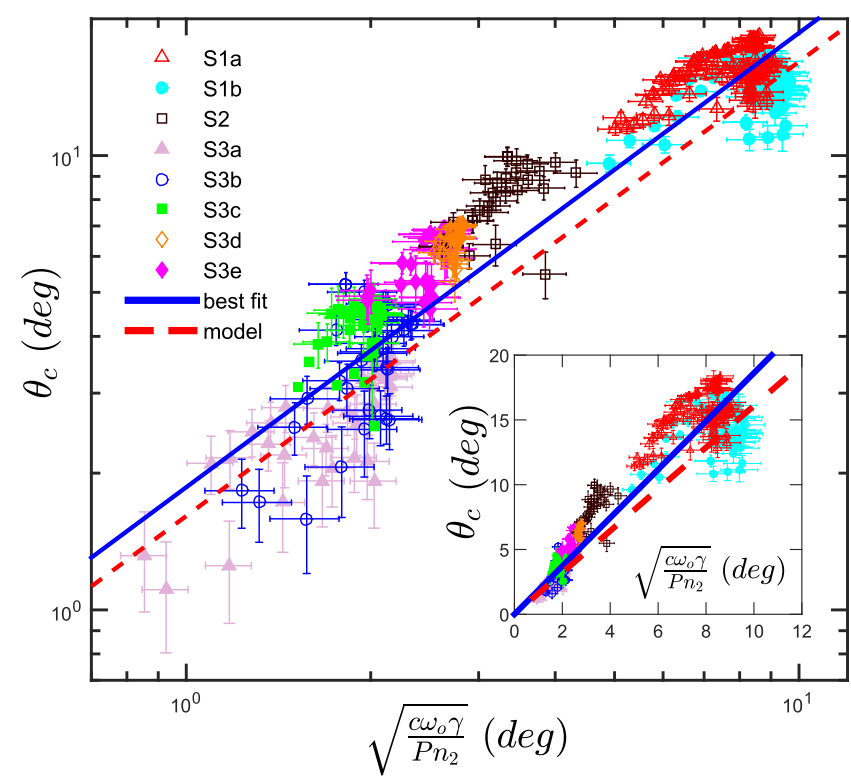

F4:1 FIG. 4. Experimental cone angles versus the characteristic cone $\mathrm{F} 4: 2$ angle $\sqrt{c w_{0} \gamma / P n_{2}}$ for all the investigated experimental systems. F4:3 The best fit is $\theta_{c}=1.86\left(c w_{0} \gamma / P n_{2}\right)^{0.5}$ whereas the dashed line F4:4 refers to Eq. (7). Inset: same plot in linear scales. (a)

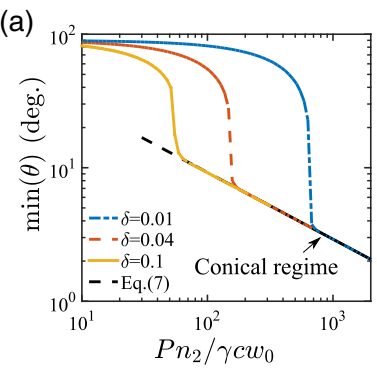

(b)

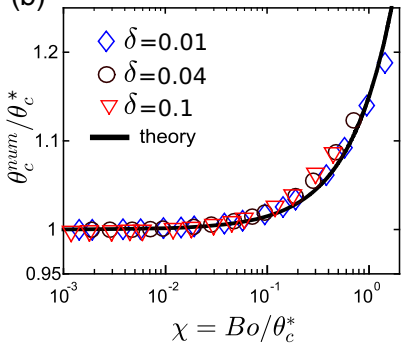

FIG. 5. (a) Theoretical minimal angle of the deformation without gravity effects for various refractive index constrast. (b) Rescaled cone angle $\theta^{\text {num }} / \theta_{c}^{*}$ numerically obtained compared with the perturbative result $\theta=\theta_{c}^{*}(1+\nu \chi)$ as a function of the $\chi=B o / \theta_{c}^{*}$ parameter for $P / P_{c}=1.35$.

F5:1

F5:2

F5:3

F5:4

F5:5

angles for all the systems at a given ratio $w_{0} / P$. Remarkably, the model is also in good agreement with the experimental data over almost two decades in interfacial tension. This strongly supports that the characteristic cone angle is given by $\sqrt{c w_{0} \gamma / P n_{2}}$ as suggested by Eq. (7).

To further confirm this model, we plot in Fig. 4 the cone semiangle $\theta_{c}$ for the eight experimental systems investigated here for all the experimental conditions as a function of the characteristic cone angle $\sqrt{c w_{0} \gamma / P n_{2}}$. Over more than one decade (see also the same data in linear scale in the inset of Fig. 4), all the data collapse into a single master curve despite some inherent dispersion of data, in particular close to the critical power $P_{c}$ where the interface sensitivity to excitation is the largest. The best fit is $\theta_{c}=$ $1.86 \sqrt{c w_{0} \gamma / P n_{2}}$, which is very close to the model prediction $\theta_{c}=1.61 \sqrt{c w_{0} \gamma / P n_{2}}$ [see Eq. (7)]. The agreement is even reinforced considering that no adjustable parameter is used in the model. Consequently, conical deformations can be fully controlled with both fluid properties and excitation parameters.

Counterintuitively, the model predicts that the cone angle $\theta_{c}$ does not depend on the relative index contrast $\delta$ [see Eq. (7)]. Indeed, as shown in Fig. 5(a), as soon as the critical power is reached, the minimal deformation angle $\min (\theta)$ switches to a single behavior independent of $\delta$. This is due to the independence of the radiation pressure with the refractive index contrast in the total reflection regime [see Eq. (5)], as opposed to the normal incidence case. However, the refractive index contrast $\Delta n$ remains essential to set the critical power $P_{c}$ to observe a cone.

We now investigate whether gravity effects could be responsible for deviations between experimental data and theory. Gravitational effects can be evaluated by forming the ratio between the buoyancy $\Delta \rho g h$ and the characteristic Laplace pressure $\gamma / w_{0}$. Since $h \sim w_{0} / \theta_{c}^{*}$ in the conical region, the relevant dimensionless parameter is $\chi=$ $\Delta \rho g w_{0}^{2} /\left(\gamma \theta_{c}^{*}\right)=B o / \theta_{c}^{*}$. Intuitively, increasing gravitational effects should flatten the deformation and thus increase the cone angle $\theta_{c}$. As explained in the Supplemental Material [24] by a perturbation analysis, the cone semiangle is 
expected to vary as $\theta=\theta_{c}^{*}(1+\nu \chi)$, where $\nu \simeq 0.14-0.18$ is a weakly varying parameter. These results are confirmed in Fig. 5(b) by comparing with the complete numerical resolution of the force balance equation [Eq. (1)] for various index ratio. The numerical results in Fig. 5(b) collapse into a single master curve, validating this perturbation analysis. We evaluate the deviations from the analytical results without gravity [Eq. (7)] to be at most $30 \%$ for the largest values of $\chi$ in our experiments $\left(\chi \approx 10^{-2}-2\right)$, confirming that gravity can be neglected at leading order.

To conclude, we have experimentally and theoretically demonstrated the existence of optically induced conical deformations. The cone morphology is controlled by the fluid properties and laser parameters. The analytical and numerical analyses quantitatively predict an optical cone semiangle in good agreement with measurements over a wide range of parameters for several liquid systems. Such cones can be considered as "optical analogues" of Taylor cones, in the sense that the structure of the electromagnetic field near the interface results from its interference with refracted ray and is strongly coupled to its deformation due to total reflection conditions. As already demonstrated for Taylor cones [7], we anticipate that the properties of these static optical cones will be a key parameter to control the hydrodynamic jet at its tip as suggested by Fig. 1(g). Our results quantitatively establish the first step towards optospraying and a new optical control of interfacial properties and interfacial morphologies. This Letter also advances a new example showing that conical shapes corresponds to a universal form when liquid interfaces are stretched beyond linearity [13].

1 The authors acknowledge financial support from CNRS, University of Bordeaux, Region Nouvelle Aquitaine (Project No. OPTORHEO 2015-1R10102-0000519) and Agence Nationale pour la Recherche ANR (Project No. FISICS ANR-15-CE30-0015-01). The authors thank Romain Pascalie and Antoine Descamps-Duval for their contributions to the experiments, Hamid Kellay and Etienne Brasselet for helpful discussions and the LOMA mechanical and electronic workshop for their technical contributions to this project.

[1] J. Zeleny, Phys. Rev. 3, 69 (1914).

[2] J. Zeleny, Phys. Rev. 10, 1 (1917).

[3] J. Fernández de La Mora, Annu. Rev. Fluid Mech. 39, 217 (2007).
[4] A. L. Yarin, S. Koombhongse, and D. H. Reneker, J. Appl. Phys. 90, 4836 (2001).

[5] J. Eggers and E. Villermaux, Rep. Prog. Phys. 71, 036601 (2008).

[6] G. I. Taylor, Proc. R. Soc. A 280, 383 (1964).

[7] A. M. Ganan-Calvo, Phys. Rev. Lett. 79, 217 (1997).

[8] R. T. Collins, J. J. Jones, M. T. Harris, and O. A. Basaran, Nat. Phys. 4, 149 (2008).

[9] S. Matsui and Y. Ochiai, Nanotechnology 7, 247 (1996).

[10] I. G. Loscertales, A. Barrero, I. Guerrero, R. Cortijo, M. Marquez, and A. Ganan-Calvo, Science 295, 1695 (2002).

[11] H. A. Stone, J. R. Lister, and M. P. Brenner, Proc. R. Soc. A 455, 329 (1999).

[12] N. Chetwani, S. Maheshwari, and H.-C. Chang, Phys. Rev. Lett. 101, 204501 (2008).

[13] G. Taylor, Applied Mechanics (Springer, 1966).

[14] I. Cohen, M. P. Brenner, J. Eggers, and S. R. Nagel, Phys. Rev. Lett. 83, 1147 (1999).

[15] J. Fernandez and G. Homsy, Phys. Fluids 16, 2548 (2004).

[16] B. W. Zeff, B. Kleber, J. Fineberg, and D. P. Lathrop, Nature (London) 403, 401 (2000).

[17] D. Baigl, Lab Chip 12, 3637 (2012).

[18] A. Author, Lab Chip 8, 1856 (2008).

[19] J.-Z. Zhang and R. K. Chang, Opt. Lett. 13, 916 (1988).

[20] H. Chraibi, D. Lasseux, E. Arquis, R. Wunenburger, and J.-P. Delville, Phys. Rev. E 77, 066706 (2008).

[21] A. Casner and J.-P. Delville, Phys. Rev. Lett. 90, 144503 (2003).

[22] A. Casner and J.-P. Delville, Phys. Rev. Lett. 87, 054503 (2001).

[23] N. G. Astrath, L. C. Malacarne, M. L. Baesso, G. V. Lukasievicz, and S.E. Bialkowski, Nat. Commun. 5, 4363 (2014).

[24] See Supplemental Material at http://link.aps.org/ supplemental/10.1103/PhysRevLett.000.000000 which includes Refs. [25-28], where we provide details on the experimental setup, the fluid parameters and the theoretical analysis.

[25] A. Pouchelon, J. Meunier, D. Langevin, D. Chatenay, and A. Cazabat, Chem. Phys. Lett. 76, 277 (1980).

[26] R. Aveyard, B. P. Binks, S. Clark, and J. Mead, J. Chem. Soc., Faraday Trans. 1 82, 125 (1986).

[27] J. Petit, D. Rivière, H. Kellay, and J.-P. Delville, Proc. Natl. Acad. Sci. U.S.A. 201207634 (2012).

[28] J. Hadamard and C. R. Hebd, Seances Acad. Sci. Paris 152, 1735 (1911).

[29] M. Tjahjadi, J. M. Ottino, and H. A. Stone, AIChE J. 40, 385 (1994).

[30] R. Wunenburger, A. Casner, and J.-P. Delville, Phys. Rev. E 73, 036314 (2006).

[31] H. Chraibi, D. Lasseux, E. Arquis, R. Wunenburger, and J.-P. Delville, Eur. J. Mech. B 27, 419 (2008). 